\title{
The Search for the Elusive Companion of EG Andromedae
}

\author{
Joseph E. Pesce and Robert E. Stencel \\ Center for Astrophysics and Space Astronomy \\ University of Colorado \\ Boulder, CO 80309-0391 USA \\ and \\ Nancy A. Oliversen \\ Computer Sciences Corporation \\ Greenbelt, Maryland USA
}

\begin{abstract}
We report observations at opposite quadratures of the interacting symbiotic binary EG Andromedae (HD 4174, Period $=470^{d}$ ). Correcting for absolute motion at the system, it appears that many of the nebular lines arise from material that moves with the red giant star. The He II feature appears to track the hot component. It may be possible to use this feature in other, similar systems in order to "pin-down" the mass ratio.
\end{abstract}

The optical spectrum of EG Andromedae was first noted by Wilson (1950) to consist of a high velocity $\left(\mathrm{v}_{r}=-96 \mathrm{~km} \mathrm{~s}^{-1}\right.$ : Oliversen et al, 1985) $\mathrm{M} 2$ giant upon which were superposed the optical emission lines of $O$ III] and the Balmer series. The ultraviolet spectrum exhibits a far UV continuum and emission lines of C IV, He II, O III] and other species. The companion star is thought to be similar to the central star of a planetary nebula (Kenyon 1983). Oliversen et al (1985) have used the spectroscopic motion of the red giant features and an estimate of the mass of the hot object $(0.7 \mathrm{M} \odot)$ to approximate the mass ratio of about 3.5 .

Oliversen et al report that the red giant optical absorption features undergo an estimated $15 \mathrm{~km} \mathrm{~s}^{-1}$ total velocity excursion between orbital quadratures. With a mass ratio of 3.5 , we predict that the hot object should exhibit $53 \mathrm{~km} \mathrm{~s}^{-1}$ in spectral line displacement. The motion of such spectral features should be easily measured given the $\pm 3 \mathrm{~km}$ $\mathrm{s}^{-1}$ precision possible with high-resolution IUE data (Ayres 1985).

Two high-dispersion spectra of EG And were obtained with the International Ultraviolet Explorer satellite SWP camera (1150-2000 $\stackrel{\circ}{A})$ (IUE: Boggess et al. 1978). The first (SWP 23692) was obtained at phase 0.73 (on the Oliversen et al. ephemeris), near maximum red giant orbital redshift. The second (SWP 26268) was approximately one half period later at phase 0.40 (maximum red giant blueshift). Both were 345 minute exposures taken on 15/16 August 1984, and 27 June 1985, respectively. After each stellar observation, 
a two minute "wavcal" Pt-Ne emission line spectrum was obtained to provide individual wavelength calibration.

Measurements of the central wavelengths and fluxes of the emission lines were obtained by fitting a least-squares Gaussian profile to the unsaturated portions of the profiles of unblended emission lines. The formal measurement error is typically $\pm 0.015 \AA$ for well exposed emission lines, except He II $( \pm 0.060 \AA)$ and O I] $( \pm 0.018 \AA)$.

Comparisons of the two corrected stellar spectra were made and the shift in wavelength between the two phases was thus obtained. The greatest velocity shift is for 0 IV] which corresponds to $+15 \mathrm{~km} \mathrm{~s}^{-1}$. Surprisingly, this is the same as the expected velocity sign and amplitude of the red giant primary. Rather than finding the $-53 \mathrm{~km} \mathrm{~s}^{-1}$ wavelength shift between quadratures for material near the hot companion, we found the majority of the UV emission lines seem to arise from material near the primary $(\mathrm{v}=+15$ $\mathrm{km} \mathrm{s}^{-1}$ ). In particular, all of the intercombination lines show a +7 to $+15 \mathrm{~km} \mathrm{~s}^{-1}$ displacement between quadratures. The resonance lines of Si IV and C IV, however, show a much smaller shift, +1 to $+6 \mathrm{~km} \mathrm{~s}^{-1}$. He II is the only feature possibly showing a systematic shift with the companion star's velocity sign $\left(-2.2 \mathrm{~km} \mathrm{~s}^{-1}\right)$ but at an amplitude many times less than expected (additional details in Pesce et al., 1987).

We propose the following picture: The intercombination lines arise from a large fraction of the red giant's outer atmosphere that is radiatively heated by the companion star and caused to expand so that part of it overflows the tidal radius and forms a stream directed at the companion. The resonance lines arise from this stream, but from material on the red giant side of the system's inner Lagrangian point, $\mathrm{L}_{1}$. The He II feature may be associated with the stream and/or accretion on the companion star's side of $L_{1}$. He II can possibly be used to track the hot component in symbiotic stars. Future studies of EG And and other systems showing He II emission are planned in order to prove this hypothesis.

Associating the resonance and intercombination lines with the red giant atmosphere supports the analysis by Friedjung, Stencel and Viotti (1983) that the red giant wind plays a major role in the spectrum formation in symbiotic binaries. Also, we are unable to verify the mass ratio of 3.5 deduced by Oliversen et al (1985), because the only feature that can be possibly associated with the motion of the hot component, He II, is mutilated by complex absorption patterns possibly associated with a stream and accretion.

\section{REFERENCES}

Ayres, T. et al. 1985 in The Future of UV Astronomy, ed., J. Mead, R. Chapman \& Y. Kondo, NASA Conf. Publ. 2349, p.468.

Boggess, A., et_al 1978 Nature 275, 1.

Friedjung, M., Stencel, R.E., and Viotti, R. $1983 A \& A$ 126, 407.

Kenyon, S.J. 1983, Ph.D.thesis, University of Illinois.

Oliversen, N.A., Anderson, C.M., Stencel, R.E., and Slovak, M.H. 1985, Ap.J. 295, 620.

Pesce, J.E., Stencel, R.E., and Oliversen, N.A. 1987, Pub.A.S.P. in press (Nov.).

Wilson, R.E. 1950, Pub.A.S.P. 62, 14. 